Math. Z. 136, 93-94 (1974)

(C) by Springer-Verlag 1974

\title{
Berichtigung zu der Arbeit: „Über die Hölderstetigkeit der schwachen Lösungen gewisser semilinearer elliptischer Systeme“
}

Math. Zeitschr. 130, $149-157$ (1973)

\section{Wolf von Wahl}

Auf S. 149 sind die Zeilen 19 von unten bis 16 von unten zu berichtigen. Es muß heißen:

oder 2. von der Gestalt $f^{l}(x, \mathbf{u}, \nabla \mathbf{u})=h^{l}(x) f^{l}(x, \mathbf{u}, \nabla \mathbf{u})$ sein, wobei $f^{l} \geqq 0$ ist, $f^{l} / u^{l}$ von quadratischem Wachstum bezüglich $\nabla \mathbf{u}$ ist und $h^{l}$ eine in $\Omega$ beschränkte meßbare Funktion bedeutet. Wir zeigen dann, ohne bei 1. a-priori eine ..."

S. 151, 4.Z.v.o.: Ersetze 2 durch -2 .

S. 151, 6.Z.v.o.: Ergänze: $\gamma>m$.

S. 152, ab 8.Z.v.u.: Ersetze $l$ durch $\tilde{l}$.

Die Argumentation auf S. 153 unten ist nicht korrekt. Vielmehr muß

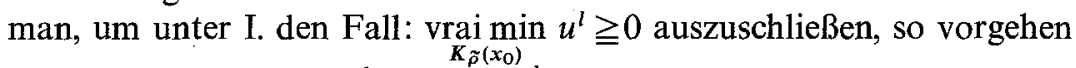
wie auf S. 154 und für $\phi_{+}^{l}$ (u) bzw. $\phi_{-}^{l}$ (u) die Funktionen

$$
\begin{aligned}
& \phi_{+}^{l}(\mathbf{u})=10 m M\left(u^{l}-\tilde{\alpha}\right)+\sum_{\tilde{i}=1}^{m}\left(u^{\tilde{l}}\right)^{2}, \\
& \phi_{-}^{l}(\mathbf{u})=10 m M\left(1-\left(u^{l}-\tilde{\alpha}\right)\right)+\sum_{\tilde{l}=1}^{m}\left(u^{\tilde{l}}\right)^{2}
\end{aligned}
$$

wählen $\left(\tilde{\alpha}:=\sup _{\tilde{\rho} \leqq \rho} \operatorname{vrai~min}_{K_{\tilde{\rho}}\left(x_{0}\right)} u^{l}\right)$.

S. 154, 10.Z.v.o.: Es muß heißen: $\phi_{+}^{l}(\mathbf{u})=10 m M\left(l-u^{l}\right)^{\dagger}+v$.

S. 154, 11.Z.v.u.: Es muß heißen: $\left(\phi_{+}^{l}(\mathbf{u})\right)_{x_{i}}$.

S. 155, ab 2.Z.v.u.: Es muß heißen: Wie in [3], S. 15-16, zeigt man, $\mathrm{da}$

$$
\int_{\tilde{\rho}\left(x_{0}\right)}|\nabla \mathbf{u}|^{2} d x \leqq c\left(\mu, \nu, \tilde{\mu},\|\mathbf{u}\|_{\left(L^{\infty}(\Omega)\right)^{m}},\left\|\mathbf{v}_{1}\right\|_{(L(\Omega))^{m}}\right) \frac{\tilde{\rho}^{2 \tilde{\alpha}}}{\rho_{1}^{* 2 \tilde{\alpha}}}
$$


mit einem Exponenten $\tilde{\alpha}, 0<\tilde{\alpha}=\tilde{\alpha}\left(\mu, v,\left\|\mathbf{v}_{1}\right\|_{\left.\left(L^{\infty}(\Omega)\right)^{m}\right)}\right)<1$, wenn $n=2$ ist. Nach dem bekannten Höldertest von Morrey folgt hieraus in der Tat Satz 1 für $n=2$. Für $n \geqq 2$ s. auch [2], Kap. VIII, § 2 .

S. 156, 3.Z.v.o.: Es muß heißen: $a_{i j}$ statt $a_{i j}^{l}$.

S. 156, 8.Z.v.o. bis 13.Z.v.o.: Es muß heißen:

$$
\left|\tilde{f}^{l}(x, \mathbf{u}, \mathbf{p}) / u^{l}\right| \leqq \tilde{\mu}(|\mathbf{u}|)\left(|\mathbf{p}|^{2}+2\right), \quad(x, \mathbf{u}, \mathbf{p}) \in \Omega \times \mathbb{R}^{m} \times \mathbb{R}^{n m},
$$

genügt, und für die $h^{l} \tilde{f}^{l} \geqq 0$ ist. Weiter seien die $h^{l}$ beschränkt und meßbar. - Dann gilt der

S. 156: Bei Satz 2 muß man voraussetzen, daß $\mathbf{u} \geqq 0$ und

$$
\underset{\substack{x \in \Omega, l \leqq l \leqq m}}{\operatorname{vrai} \max }\left|h^{l}(x)\right| \tilde{\mu}\left(\|\mathbf{u}\|_{\left(L^{\infty}(\Omega)\right)^{m}}\right)\|\mathbf{u}\|_{\left(L^{\infty}(\Omega)\right)^{m}}^{2} \leqq \frac{\tilde{v}}{m}
$$

ist mit einem $\tilde{v}<v$. Die Abhängigkeit von $c$ vom Stetigkeitsmodul der $h^{l}$ entfällt.

S. 156, ab 10.Z.v.u.: Es muß heißen:

Beweis. Sei $K_{\rho}\left(x_{0}\right)$ ein kompakt in $\Omega$ enthaltene Kugel vom Radius $\rho$. Dann ist

$$
\int_{K_{\rho}\left(x_{0}\right)} \sum_{i, j=1}^{n} a_{i j}(x) u_{x_{i}}^{l} \eta_{x_{j}}^{l} d x \leqq 0, \quad 1 \leqq l \leqq m, \eta^{l} \in \stackrel{H}{ }^{1}\left(K_{\rho}\left(x_{0}\right)\right), \eta^{l} \geqq 0,
$$

und

$$
\left|\widetilde{K}_{\widetilde{K}_{\rho}\left(x_{0}\right)} \sum_{l=1}^{m} f^{l}(x, \mathbf{u}, \mathbf{\nabla u}) \frac{\zeta^{2}}{u^{l}+\varepsilon} d x\right| \leqq \tilde{v} \sum_{l=1}^{m} \int_{K_{\rho}\left(x_{0}\right)}\left|\nabla u^{l}\right|^{2} \zeta^{2} \frac{d x}{\left(u^{l}+\varepsilon\right)^{2}}
$$

für alle hinreichend kleinen $\varepsilon$ und alle lipschitzstetigen $\zeta$, die auf dem Rand von $K_{\rho}\left(x_{0}\right)$ verschwinden. Wie in [2], S. 426-427, folgt hieraus der Satz, wenn man statt $\xi$ auf S. 427 die Funktionen

$$
\xi_{l}(x)=\zeta^{2}(x) \cdot v_{l}(x) \cdot \max \left\{\sum_{i=1}^{n} v_{i}^{2}-k, 0\right\},
$$

$1 \leqq l \leqq m$, wählt, wobei $v_{l}$ in jeder Zeile statt $v$ steht.

\author{
Prof. Wolf v. Wahl \\ Math. Institut der Universität Bochum \\ D-4630 Bochum \\ Universitätsstr. 150 \\ Bundesrepublik. Deutschland
}

